\title{
Work in Progress: Creating an Engineering-based Medical School to Address a Critical Gap in Medical Innovation
}

\section{Dr. Jennifer R Amos, University of Illinois, Urbana-Champaign}

Dr Amos joined the Bioengineering Department at the University of Illinois in 2009 and is currently a Teaching Associate Professor in Bioengineering and an Adjunct Associate Professor in Educational Psychology. She received her B.S. in Chemical Engineering at Texas Tech and Ph.D. in Chemical Engineering from University of South Carolina. She completed a Fulbright Program at Ecole Centrale de Lille in France to benchmark and help create a new hybrid masters program combining medicine and engineering and also has led multiple curricular initiative in Bioengineering and the College of Engineering on several NSF funded projects.

\section{Gabriella R Dupont, University of Illinois, Urbana-Champaign}

I am a MEng student in Bioengineering, with a BS, Bioengineering, both from University of Illinois, Urbana-Champaign. I am interested in biomechanics and how curriculum structure affects education outcomes. 


\section{Creating an Engineering-based medical school to address a critical gap in medical innovation}

\section{Introduction}

Healthcare and medicine will change dramatically in response to external factors such as inequities driven by rising costs of healthcare, the role of technology in medicine, and ethical dilemmas driven by increases in population and age-related diseases [1]. To anticipate and drive these changes, our students must not only possess the technical abilities to solve these problems, but must have the contextual and leadership skills to create solutions.

The University of Illinois at Urbana-Champaign has acted a regional site for a medical school for over 50 years. As a regional site, our campus educated 125 first year medical students in basic sciences education, typically known as the M1 curriculum. Out of those 125, 25 students stayed on our campus for M2 -M4 and our local hospital hosts clerkships and clinical electives for those students. This regional site also offered a combined $\mathrm{MD} / \mathrm{PhD}$ option, which allows students admitted to both campuses to pursue both degrees simultaneously. According to historical admissions tracking in the local $\mathrm{MD} / \mathrm{PhD}$ program, approximately $25 \%$ of the $\mathrm{MD} / \mathrm{PhD}$ students are pursuing a $\mathrm{PhD}$ in engineering, which is a contrast to a 2008 survey of more than 24 $\mathrm{MD} / \mathrm{PhD}$ programs with an average $9 \%$ enrollment in an engineering $\mathrm{PhD}$ [2]. This is likely attributed to the strength and ranking of the College of Engineering on the university campus.

Over the past year, the University of Illinois at Urbana-Champaign proposed a request for a new, independently accredited medical school on the engineering university campus [3]. The newly formed Carle Illinois College of Medicine is the first Engineering Based College of Medicine where engineering would be integrated across the entire curriculum along with clinical and basic sciences. As part of the study, we conducted interviews of current medical school professors, faculty, and students focusing on the perceptions and attitudes of medical students with engineering backgrounds. Distinct divisions between MD and MD/PhD trained students emerged through this process.

\section{Methods}

We conducted our research using the Delphi method, which consults a group of experts on the topic to obtain a consensus on a specific topic or trend [4]. The Delphi method was developed to sample a group of experts on a particular topic and gain consensus of opinion, particularly related to a new trend or prediction. As the merging of engineering and medicine is relatively new, this approach is appropriate for gaining consensus of skills, knowledge, and abilities needed in each respective field from experts in these areas [5].

The method is a three-part questionnaire aimed at finding similarities and disparities in opinions between experts. The first stage is an open questionnaire where participants provide answers to questions with as much detail as they like. The second stage involves the research team assembling individual responses to gain perspective on group views and producing the analytics to support a group view. The third phase focuses on disagreements among participants. In this phase, the researchers consult with experts on specific areas of disagreement, allowing the participant to reflect on their response compared to the group and defend or adapt their response. The final phase is to send back the homogenized views to the participants to seek consensus [5]. As opposed to interviews, surveys, and focus groups, the success of this method is linked to the 
ability of an individual to express an opinion and then revise it based on group views and efficient use of time for panels and researchers [5].

Delphi results are semi-quantitative and are analyzed by calculating medians and interquartile ranges. They are used to identify the rates of group agreement and consensus for each item that a panelist makes as a statement [6]. We conducted the study on current medical school students with engineering backgrounds, faculty of the current medical school, and faculty involved in designing a new engineering-based medical school.

We first conducted a survey on the skills, abilities, perceptions, and identities of medical students with backgrounds in engineering and collected survey responses from the medical students group. Students were recruited from the regional medical campus and from recent alumni from the bioengineering program currently enrolled in other medical schools, leading to a population size of 90 students. We had a $15 \%$ response rate to the survey, $N=14$ with 8 female and 6 male respondents.

The research team then sent surveys to a selection of faculty and staff. The participants were chosen because of their involvement with the current or future college of medicine. The population size of the invitation was 24 , with $\mathrm{N}=3$ responses. We summarized both the faculty and student opinions from survey responses and prepared an interview where we used those summaries to create questions.

Finally, we invited the same selection of faculty and staff to participate in an interview with the research team to comment on the initial findings. We asked the subjects to comment on the consensus of the group from the first survey, and then collected a consensus from those interviews. We conducted the interviews either in person or over the phone, and recorded all interviews for accuracy. The team created a transcription of the conversations and summarized them. We used the summary to create a consensus among the group. The subjects remained anonymous to the coordinator of the study throughout the process. The team conducted 9 interviews.

We took the perceptions from the interviews to a broad committee of faculty, which hoped to address the perceptions through curriculum and clerkship development.

\section{Results}

In the survey results of engineering students currently enrolled in a medical degree, $70 \%$ of students responded that they felt they have enhanced reasoning and problem-solving skills compared to non-engineering peers. $50 \%$ of respondents felt that they had weaknesses in memorization skills and biological content compared to non-engineering peers. $60 \%$ of respondents also felt that their social skills and social understanding is weaker than nonengineering peers. They cited experiences of being told they were anti-social or not understanding societal and cultural norms, which negatively affected them in the clinic. The students made suggestions for engineering and clinical integration in core classes such as Physiology and Histology and Physical Diagnosis, since those courses were most conducive to introducing engineering concepts. 
In the interviews with faculty members and clinicians, we covered three general topics: learning, communication and professional skills, and curricular changes. There was a consensus that engineers learn by doing, break down problems, and are good with analytical solutions, while they tend to have a lack of basic biology background. In terms of soft skills and patient interaction skills, the interviewees overwhelmingly said that engineers ask more questions of "why is this patient sick," not just "how do we treat this." They also said that there may be a perceived loss of humanism and less compassion for those "less intellectually gifted."

The faculty members and clinicians also offered suggested curriculum changes or additions consistent with their observations about how engineers think and behave as doctors. They emphasized team based, problem based and hands on learning and suggested an argumentation course and philosophy or religious studies, to round out the perceived shortcomings of engineerdoctors.

\section{Discussion}

Overall, there was consistency among the faculty, clinicians, and students on the perceptions of engineer-doctors. One interesting difference between the two groups was that most of the students felt that they did lack social skills and understanding, while most of the faculty interviewees mentioned that although they did have this perception, most of the students they encounter do not actually display those deficits. The faculty members that we interviewed who don't have direct interaction with engineer-doctors currently stood by their comments that the students lack the social skills or humanities background desired by MD programs. However, the faculty members who currently interact with the MD students on campus commented on the fact that they initially thought the lack of social skills would be an issue, they do not encounter this issue at all with the engineer-doctors they interact with.

One interviewee commented on professionalism, saying "Engineers were in professional schools as undergrads, so they will have innate professional ability," which speaks to the educational outcome of undergraduate programs in engineering having a focus on applicable skills for a workplace of teamwork, presentation skills, and professional conduct. Engineering schools are accredited by ABET, and are required to show that students have, "an ability to function on multidisciplinary teams... and understanding of professional and ethical responsibility... and ability to communicate effectively... a knowledge of contemporary issues... the broad education necessary to understand the impact of engineering solutions in a global, economic, environmental, and societal context" [7].

\section{Future Directions}

The results of this study will be used to advise the creation of the first engineering-based medical school. The guiding principle is that engineering is a way of thinking about problems, recognizing the underlying conservation laws that all physical objects obey, and using that to quickly and quantitatively understand complex physiological processes. The answer is not adding an engineering curriculum to the medical curriculum. Instead, one must leverage a student's previous engineering training to provide the medical curriculum in their language (quantitative with differential equations) so that they do not have to memorize qualitative relationships in physiology. Engineers can learn medicine with an enduring understanding of quantitative relationships and functions of the human body. 
The infusion of engineering aims to capture quantitative relationships to elucidate the behavior and alleviate the need for memorizing links between variables. As an example: blood pressure is a combination of cardiac output, arterial compliance, and peripheral resistance. In many traditional physiology texts, plots are used to demonstrate the relationship between compliance and changes in aging vasculature and blood pressure, resulting in a qualitative understanding of the relationship. However, for the engineer, a simple first-order differential equation can be used to generate understanding of the relationship, $\frac{d P_{S A}}{d t}=\frac{1}{C_{S A}}\left(Q_{A o}-\frac{P_{S A}}{R_{S}}\right)$. The engineer sees a first order system with a rate constant and can understand the relationship between compliance of the artery and the pressure from the stroke volume. Due to the quantitative understanding, there are no longer qualitative relationships to be memorized.

Not only does this approach provide significant benefit in learning medical physiology concepts for students who have been trained to think like engineers, but with this quantitative knowledge, the future MD can model, predict, and simulate pathology and interventions to better understand the progress of disease in an individual. Further, quantitative understanding and modeling requires parameters and units on measures, tying this level of understanding to the measurement systems required to discover a particular patient's parameters.

We can change the medical education system to embrace these skills, including problem-based learning trends observed at other medical schools [8] and multidisciplinary teamwork between the engineers and social science background students. This will result in better innovators, doctors, and researchers who are more able and interested in addressing the growing healthcare needs as the population ages.

\section{Citations}

1. Cassel C. Better Health care and Lower Costs: Accelerating Improvement Through Systems Engineering. Report to the President, Executive Office of the President.

2. Brass LF, Akabas MH, Burnley LD, Engman DM, Wiley CA, Andersen OS. Are MD$\mathrm{PhD}$ programs meeting their goals? An analysis of career choices made by graduates of $24 \mathrm{MD}-\mathrm{PhD}$ programs. Academic medicine: journal of the Association of American Medical Colleges. 2010 Apr;85(4):692.

3. Chan LS. Building an Engineering-Based Medical College: Is the Timing Ripe for the Picking?. Medical Science Educator. 2016 Mar 1;26(1):185-90.

4. Dalkey N, Helmer O. An experimental application of the Delphi method to the use of experts. Management science. 1963 Apr;9(3):458-67.

5. Hsu, C. C., \& Sandford, B. A. (2007). The Delphi technique: making sense of consensus. Practical Assessment, Research \& Evaluation, 12(10), 1-8.

6. Fish, L. S., \& Busby, D. M. (1996). The Delphi method. Research methods in family therapy, 469-482.

7. Criteria for accrediting engineering programs, 2016-2017. ABET. http://www.abet.org/accreditation/accreditation-criteria/criteria-for-accreditingengineering-programs-2016-2017/\#outcomes Accessed 21 Sep 2017.

8. Kaufman, D., Sutow, E., \& Dunn, K. (2017). Three approaches to cooperative learning in higher education. Canadian Journal of Higher Education, 27(2/3), 37-66. 
